\title{
Aspiration cytology of an ectopic cervical thymoma misinterpreted as a lymphoproliferative lesion of the thyroid: A case report
}

\author{
YI-YING LEE ${ }^{1}$, WEN-CHING WANG ${ }^{2}$ and CHIEN-FENG LI ${ }^{1,3-5}$ \\ Departments of ${ }^{1}$ Pathology and ${ }^{2}$ General Surgery, Chi-Mei Medical Center, Tainan 71004; \\ ${ }^{3}$ National Institute of Cancer Research, National Health Research Institutes, Tainan 71005; \\ ${ }^{4}$ Kaohsiung Medical University, Kaohsiung 80708; ${ }^{5}$ Department of Biotechnology, \\ Southern Taiwan University of Science and Technology, Tainan 71005, Taiwan, R.O.C.
}

Received June 17, 2014; Accepted February 26, 2015

DOI: $10.3892 / 01.2015 .3423$

\begin{abstract}
Ectopic cervical thymoma is a rare tumor that originates from ectopic thymic tissue trapped during the migration of the embryonic thymus. To the best of our knowledge, only 14 cases of ectopic cervical thymoma, which include descriptions of the cytological features based on fine-needle aspiration (FNA), have been reported thus far. The current study describes the case of a 52-year-old male presenting with an enlarging anterior neck mass that been apparent for a number of years and was now accompanied by shortness of breath. FNA cytology revealed large numbers of small lymphocytes admixed with rare groups of large, polygonal cells that were interpreted to be reactive lymphocytes or possible follicular dendritic cells. However, no definite follicular or Hürthle cells were identified. Therefore, the overall cytological features were misinterpreted as a lymphoproliferative lesion. However, subsequent histological analysis of the resected left total lobectomy specimen determined a diagnosis of thymoma, type B1. Thus, awareness of this entity combined with a careful search for thymic epithelial cells may aid in determining a correct diagnosis when FNA is performed for the evaluation of a neck mass.
\end{abstract}

\section{Introduction}

Ectopic thymoma is a rare tumor that originates from ectopic thymic tissue trapped during the migration of the embryonic thymus (1). The incidence of ectopic thymoma is $\sim 4 \%$ (2). Various locations of ectopic thymoma have been reported, with the cervical region (ectopic cervical thymoma) appearing to be one of the most frequent. Ectopic cervical thymoma commonly occurs in the anterolateral region of the neck, or subjacent to or

Correspondence to: Dr Chien-Feng Li, Department of Pathology, Chi-Mei Medical Center, 901 Chunghwa Road, Tainan 71004, Taiwan, R.O.C.

E-mail: angelo.p@yahoo.com.tw

Key words: ectopic cervical thymoma, fine-needle aspiration, neck inside the lower pole of the thyroid gland (1-5). Furthermore, ectopic cervical thymoma commonly presents as a palpable neck mass that can be easily examined by fine-needle aspiration (FNA). However, due to its unusual locations and rare incidence, it is easy to misinterpret the cytological features of an ectopic cervical thymoma as a benign or malignant thyroid lesion, such as thyroiditis, a thyroid malignancy or malignant lymphoma of the thyroid (3-5). The present study reports a case of this rare entity that was misdiagnosed as a lymphoproliferative lesion by FNA.

\section{Case report}

In December 2012, a 52-year-old male presented to Chi-Mei Medical Center (Tainan, Taiwan) with a five-year history of a gradually enlarging anterior neck mass that had recently caused shortness of breath. Laboratory examinations, including blood count, biochemistry and serum thyroglobulin levels, were within normal limits. However, neck MRI revealed a $6.4 \times 4.8 \times 4.4-\mathrm{cm}$ mass in the left thyroid accompanied by tracheal deviation, indicating a nodular goiter (Fig. 1A).

Ultrasonography-guided FNA was performed, yielding abundant small- to medium-sized lymphocytes mixed with scant larger cells with round to oval nuclei and marginally more abundant cytoplasm (Fig. 1B). These larger cells were interpreted to be reactive lymphocytes or possible follicular dendritic cells. However, no follicular cells, specifically Hürthle cells, were identified. Therefore, the mass was diagnosed as a lymphoproliferative lesion.

A subsequent left total thyroidectomy with mass excision was performed as a result of the FNA smears. The tumor was located external to the left lobe of the thyroid, but attached to the lower end. The $6.5 \times 4 \times 3.5-\mathrm{cm}$ excised tumor $(22.5 \mathrm{~g})$ was encapsulated, with a whitish-gray and lobulated cut surface (Fig. 1C), and was easily separated from the grossly normal $4 \times 3 \times 2 \mathrm{~cm}$ thyroid $(23.5 \mathrm{~g})$. The resected tissue was fixed in formalin and embedded in paraffin, prior to $4-\mu \mathrm{m}$ thin sections being cut and stained with hematoxylin and eosin.

Microscopic analysis of the frozen sections revealed that the tumor was predominantly composed of a densely packed population of small lymphocytes in expansile sheets with preserved lobular architecture and separated by fibrous 
bands (Fig. 2A). In addition, the presence of a small number of interspersed large, polygonal cells characterized by round, vesicular nuclei and occasionally prominent nucleoli was focally identified (Fig. 2B). Furthermore, microcystic changes with Hassall's corpuscles were present in the subcapsular area (Fig. 2C). These features indicated the possibility of a lymphoid lesion.

Immunohistochemical analysis was performed using a labeled streptavidin-biotin peroxidase kit (Dako North America, Inc., Carpinteria, CA, USA). The antibodies used were AE1/AE3 (Dako North America, Inc.), cluster of differentiation (CD)1a, CD5, CD99 and terminal deoxynucleotidyl transferase (TdT; Novocastra Laboratories Ltd., Newcastle upon Tyne, UK) . The large polygonal cells immunohistochemically expressed AE1/AE3 (Fig. 2D). By contrast, the small lymphocytes showed immunopositivity for the immature T-cell markers, CD1a (Fig. 2E), CD5 and TdT. Thus, the neck tumor mimicking a thyroid mass was diagnosed as ectopic thymoma, type B1, according to the World Health Organization (WHO) classification (6). The adjacent non-neoplastic thyroid exhibited no abnormal pathological findings. Retrospective analysis of the FNA cytology revealed a rare groups of large cells that were positive for AE1/AE3 on destained Papanicolaou stain slides (Fig. 2F), indicating that these cells may have represented thymic epithelial cells At the six-month post-operative follow-up, the patient was healthy, with no known recurrence or distant metastasis of the tumor.

\section{Discussion}

In contrast to mediastinal thymomas, thymomas arising in ectopic cervical thymic tissue are rare and most often affect female individuals, with a female:male ratio of $7: 1(1,4,5)$. The majority of reported ectopic cervical thymomas are not associated with myasthenia gravis or other paraneoplastic manifestations; only rarely have cases been reported in association with myasthenia gravis $(7,8)$. The gross histological features of ectopic cervical thymoma are identical to its mediastinal counterparts. Furthermore, the clinical course is typically benign, with tumor recurrence or metastasis rarely observed $(9,10)$.

Although the microscopic features of ectopic and mediastinal thymoma are parallels, the rarity and unusual location of ectopic thymoma means that a straightforward diagnosis, particularly based on FNA cytology, is difficult (10). Thymoma is composed of varying proportions of neoplastic epithelial and reactive lymphoid elements, and the cytological features of the tumor differ depending on the cellular composition. When the epithelial and lymphocytic components are obvious in the aspirate, a diagnosis of thymoma can be easily attained. However, when a single component is predominant, determining an accurate diagnosis is more difficult (4).

To the best of our knowledge, only 14 cases of ectopic cervical thymoma that include descriptions of the cytological features based on FNA have been documented to date $(5,11)$. Taweevisit et al (4) compiled the details of all 14 cases in a literature review. The initial cytological diagnoses included Hashimoto thyroiditis, lymphocytic thyroiditis, papillary and medullary carcinomas, malignant lymphoma of the thyroid
$\mathbf{A}$

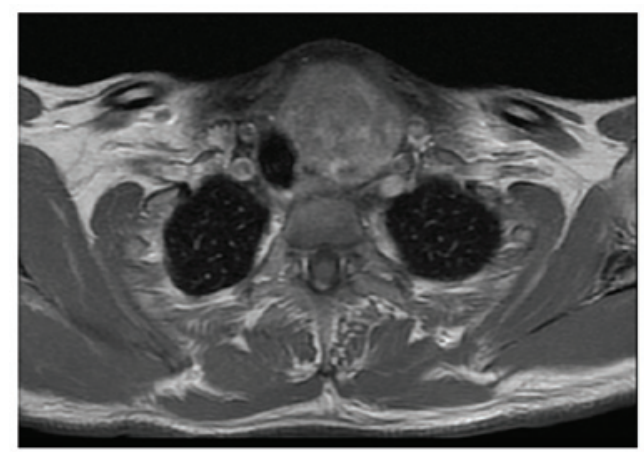

$\mathbf{B}$

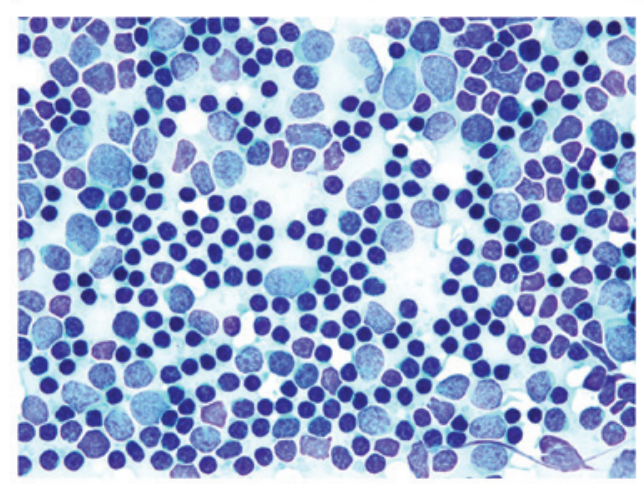

C

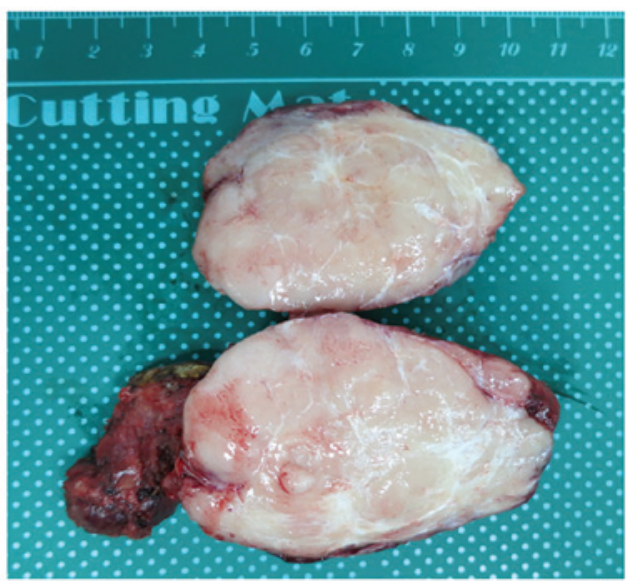

Figure 1. (A) T1-weighted magnetic resonance imaging, revealing a $6.4 \times 4.8 \times 4.4-\mathrm{cm}$ tumor with heterogenous contrast enhancement in the left thytroid, causing tracheal deviation. (B) Fine-needle aspiration smear demonstrating multiple clusters of small lymphocytes admixed with scant groups of larger cells displaying round to oval nuclei and inconspicuous nucleoli with marginally more abundant cytoplasm (Papanicolaou staining); magnification, $x 1,000$. (C) The resected tumor, exhibiting a lobulated and gray-white cut surface with an irregular border. Discernible connective tissue septa were observed and the mass was located externally, but attached to the outer surface of the lower left pole of the thyroid gland.

and various types of soft-tissue tumor (3-5,7,11-20). According to the 2004 WHO classification system, the thymoma subtypes were predominantly type $\mathrm{AB}$ and type $\mathrm{B} 1$, as well as two type A thymomas, one type B2 thymoma and one type B3 thymoma $(5,6)$.

In five of the reported cases, epithelial cells were the predominant component; the cells had a round-polygonal or spindle shape with eosinophilic cytoplasm. These tumors were misdiagnosed as primary thyroid carcinoma, metastatic carcinoma and various types of soft-tissue tumor $(5,11,13,14,16)$. In one case, cohesive epithelial cell clusters with rare intranuclear inclusions were observed, resulting in the misdiagnosis as a 

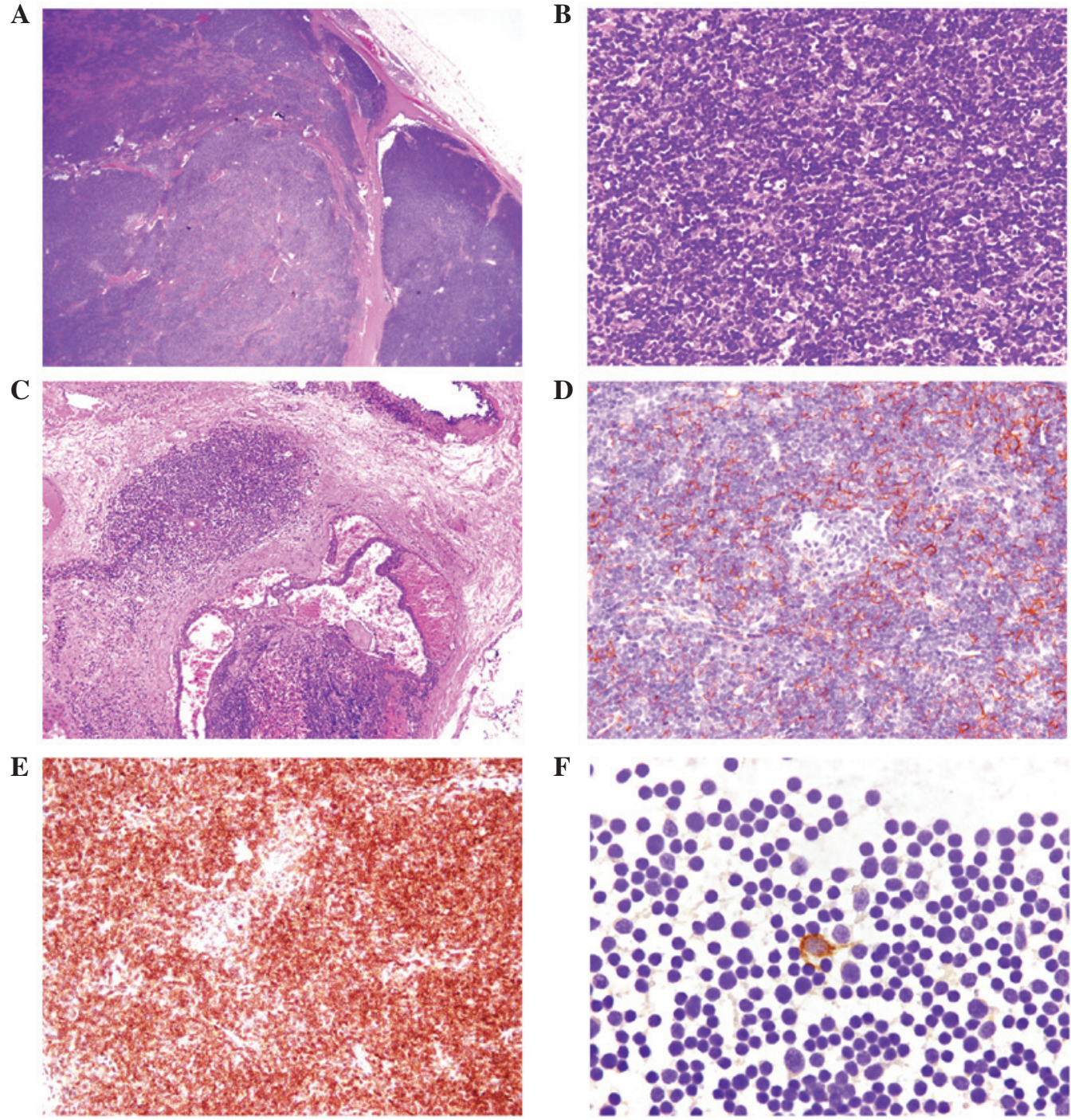

Figure 2. (A-C) Microscopic analysis of the resected tumor using hematoxylin and eosin staining. (A) Low-power view demonstrating a nodular growth pattern separated by fibrous septa; magnification, $x 40$. (B) High-power view indicating numerous small- to medium-sized lymphocytes admixed with scattered large, polygonal epithelial cells characterized by round, vesicular nuclei and occasionally prominent nucleoli; magnification, x400. (C) Residual ectopic thymic tissue with microcystic changes present in the subcapsular area; magnification, $x 40$. (D-F) Immunohistochemical analysis of the tumor using immunoperoxidase staining. (D) Lace-like pattern of epithelial cells exhibiting AE1/AE3 expression; magnification, x400. (E) Background lymphocytes expressing cluster of differentiation 1a; magnification, x400. (F) Large cells from the fine-needle aspiration smear expressing AE1/AE3; magnification, x1,000.

papillary carcinoma (16). Identifying typical cytological features, such as papillary architecture and nuclear changes, including powdery chromatin, nuclear grooves and the psammoma bodies observed in papillary thyroid carcinoma, which are absent from ectopic thymoma, may be useful for forming a differential diagnosis. For example, intranuclear inclusions and stromal amyloid do not occur in ectopic thymoma, and, thus, may be useful for distinguishing it from a spindle-cell lesion, such as a medullary carcinoma (13).

The majority of the reported cases were misdiagnosed as thyroiditis or malignant lymphoma due to a predominantly lymphoid population $(3,4,7,12,15,19)$. In such cases, the large, inconspicuous epithelial cells were misinterpreted as follicular dendritic cells or cells of undetermined nature (5). This was also true in the present case. The current patient, a 52-year-old male, presented with an enlarging mass in the anterior neck. The mass was closely associated with the thyroid gland, and clinically, the patient did not exhibit myasthenia gravis. The initial FNA diagnosis was of a reactive lymphoid population, indicating the possibility of a benign lymphoproliferative lesion. However, a retrospective review of the patient's FNA smears revealed a background of numerous small lymphocytes mixed with rare groups of large, polygonal cells that may represent neoplastic thymic epithelial cells. These large cells were positive for AE1/AE3 immunostaining, confirming their epithelial origin.

In consideration of the diagnostic course of the present case, a careful search for large, oblong- to spindle-shaped epithelial cells and the absence of Hürthle and/or follicular cells may facilitate the differential diagnosis of ectopic thymoma from thyroiditis (4). In addition, plasma cells, tangible-body macrophages, dendritic-lymphocytic aggregates and multinucleated foreign body giant cells are indicative of chronic lymphocytic (Hashimoto) thyroiditis (4). Furthermore, immunohistochemical analysis using cytokeratin and lymphoid markers, including immature T-cell markers, may be useful for 
establishing the epithelial but not lymphomatous nature of the ectopic thymoma.

In conclusion, the current study presents the clinicopathological features of a single case of ectopic cervical thymoma. A lack of awareness of this condition initially resulting in an erroneous diagnosis based on FNA cytology. Therefore, awareness of this entity, combined with a careful search for thymic epithelial cells in the aspirate and precise interpretation of all analyses, appear to be the key to forming an accurate diagnosis.

\section{Acknowledgements}

The authors appreciate the support of the Biobank of Chi Mei Medical Center (Tainan, Taiwan).

\section{References}

1. Chan JK and Rosai J: Tumors of the neck showing thymic or related branchial pouch differentiation: a unifying concept. Hum Pathol 22: 349-367, 1991.

2. Amodeo G, Cipriani O, Orsini R, and Scopelliti D: A rare case of ectopic laterocervical thymoma. J Craniomaxillofac Surg 41: 7-9, 2013.

3. Yan B, Lim D and Petersson F: Ectopic cervical thymoma: a report of two cases of a rare entity frequently misdiagnosed on fine needle aspiration cytology and frozen section. Head Neck Pathol 4: 152-156, 2010.

4. Oh YL, Ko YH and Ree HJ: Aspiration cytology of ectopic cervical thymoma mimicking a thyroid mass. A case report. Acta Cytol 42: $1167-1171,1998$.

5. Taweevisit M, Sampatanukul P and Thorner PS: Ectopic thymoma can mimic benign and malignant thyroid lesions on fine needle aspiration cytology: a case report and literature review. Acta Cytol 57: 213-220, 2013.

6. Travis WD, Brambilla E, Müller-Hermelink HK and Harris CC (eds): World Health Organization Classification of Tumours. Pathology and Genetics of Tumours of the Lung, Pleura, Thymus and Heart. Third Edition. IARC Press, Lyon, 2004.
7. Choi H, Koh SH, Park MH and Kim SH: Myasthenia gravis associated with ectopic cervical thymoma. J Clin Neurosci 15: 1393-1395, 2008.

8. Wu TH, Jin JS, Huang TW, Chang H and Lee SC: Ectopic cervical thymoma in a patient with myasthenia gravis. J Cardiothorac Surg 6: 89, 2011.

9. Kinoshita T, Yoshida J, Ishii G, Aokage K, Hishida T and Nagai K: Pulmonary metastasis from encapsulated cervical ectopic type a thymoma. Ann Thorac Surg 94: e141-e142, 2012.

10. Jung JI, Kim HH, Park SH and Lee YS: Malignant ectopic thymoma in the neck: a case report. AJNR Am J Neuroradiol 20: 1747-1749, 1999 .

11. Milde P and Sidawy MK: Thymoma presenting as a palpable thyroid nodule: a pitfall in fine needle aspiration (FNA) of the neck. Cytopathology 10: 415-419, 1999.

12. Cohen JB, Troxell M, Kong CS and McDougall IR: Ectopic intrathyroidal thymoma: A case report and review. Thyroid 13: 305-308, 2003.

13. Gerhard R, Kanashiro EH, Kliemann CM, Juliano AG and Chammas MC: Fine-needle aspiration biopsy of ectopic cervical spindle-cell thymoma: A case report. Diagn Cytopathol 32: 358-362, 2005.

14. Jin XJ, Song JY, Choi SJ, et al: Aspiration cytology of cervical thymoma: A case report. Korean J Pathol 44: 444-447, 2010.

15. Martin JM, Randhawa G and Temple WJ: Cervical thymoma. Arch Pathol Lab Med 110: 354-357, 1986.

16. Matsuura B, Tokunaga H, Miyake T, Utsunomiya S, Minami H and Onji M: A case of malignant thymoma mimicking thyroid carcinoma: A pitfall in fine-needle aspiration. Endocr $\mathbf{J} 51$ : 237-241, 2004.

17. Ponder TB, Collins BT, Bee CS, Silverberg AB, Grosso LE and Dunphy CH: Diagnosis of cervical thymoma by fine needle aspiration biopsy with flow cytometry. A case report. Acta Cytol 46: 1129-1132, 2002.

18. Vannucci J, Tassi V, Monacelli M and Puma F: Totally cervical thymoma from the orthotopic thymus. Thorac Cardiovasc Surg 60: 175-176, 2012.

19. Vengrove MA, Schimmel M, Atkinson BF, Evans D and LiVolsi VA: Invasive cervical thymoma masquerading as a solitary thyroid nodule. Report of a case studied by fine needle aspiration. Acta Cytol 35: 431-433, 1991.

20. Zeppa P, Varone V, Cozzolino I, Salvatore D, Vetrani A and Palombini L: Fine needle cytology and flow cytometry of ectopic cervical thymoma: A case report. Acta Cytol 54 (Suppl 5): S998-S1002, 2010. 